\title{
Popeye's sign, heart disease, and amyloidosis
}

\author{
Gonzalo Barge-Caballero a,b, Paula Gargallo-Fernández ${ }^{\mathrm{c}}$, Eduardo Barge-Caballero ${ }^{\mathrm{a}, \mathrm{b}}$, \\ María G Crespo-Leiro,
}

\begin{abstract}
${ }^{a}$ Advanced Heart Failure and Heart Transplant Unit, Complexo Hospitalario, Universitario A Coruña, Instituto de Investigación Biomédica de A Coruña, A Coruña, Spain; ${ }^{b}$ Centro de Investigación Biomédica en Red Cardiovascular, Instituto de Salud Carlos III, Madrid, Spain; ${ }^{c}$ Department of Cardiology, Complexo Hospitalario Universitario de Pontevedra, Pontevedra, Spain
\end{abstract}

A 69-year-old man presented to our cardiology clinic troubled by breathlessness on exertion. He had a history of hypertension, atrial fibrillation, and bilateral carpal tunnel syndrome. Physical examination showed jugular venous distension, bilateral ankle oedema, and a bunching of the right biceps when he flexed his arm-so called Popeye's sign-indicating rupture of the proximal biceps tendon (figure and video); a rupture was confirmed by an ultrasound scan. Serum N-terminal-pro-B-type natriuretic peptide was $7088 \mathrm{pg} / \mathrm{mL}$ (normal range for patients aged 0-74 years is less than $125 \mathrm{pg} / \mathrm{mL}$ ). A 12-lead electrocardiogram showed low voltage in the limb leads, as well as a pseudo-infarct pattern in the precordial ones (appendix). A transthoracic echocardiogram showed left ventricular hypertrophy with a maximum wall thickness of $20 \mathrm{~mm}$ and a left ventricular ejection fraction (LVEF) of $40 \%$. Cardiac MRI showed signs of myocardial infiltration. ${ }^{99 \mathrm{~m}}$ Technetium-3,3-diphosphono-1,2-propanodicarboxylic acid $\left({ }^{99 \mathrm{~m}} \mathrm{Tc}-\mathrm{DPD}\right)$ scintigraphy showed grade 3 myocardial uptake (figure). Serum and urine immunoelectrophoresis and free light chain assays ruled out a monoclonal component. Genetic sequencing analysis of the transthyretin gene found no mutations. Tying all the findings together, a diagnosis of heart failure secondary to wild-type transthyretin amyloid (ATTRwt) cardiomyopathy was made. The patient was treated with a combination of diuretics - namely, furosemide $80 \mathrm{mg}$ twice a day, hydrochlorothiazide $12 \cdot 5 \mathrm{mg}$ once a day, and spironolactone $100 \mathrm{mg}$ once a day-but he did not respond well. He remained symptomatic with advanced functional impairment and persistent signs of systemic venous congestion.

ATTRwt, previously known as senile systemic amyloidosis, is a disease that typically affects the heart and tendons of elderly patients. A history of bilateral carpal tunnel syndrome or, less frequently, spontaneous tendon rupture in a patient with heart failure and preserved or mildly depressed LVEF should raise clinical suspicion of the condition. A definitive diagnosis of ATTRwt cardiomyopathy can be confirmed non-invasively with a positive finding on ${ }^{99 \mathrm{~m}} \mathrm{Tc}-\mathrm{DPD}$ scintigraphy, together with the absence of a monoclonal component in serum and urine samples, and a normal genetic sequencing analysis of the transthyretin gene. Current therapy for patients with ATTRwt cardiomyopathy is limited, with diuretics for relief of cardiac congestion being the mainstay of treatment. However, a recent randomised, placebocontrolled trial, published in September 2018, showed a significant reduction in the risk of death and hospitalisation from cardiovascular causes in patients with ATTRwt cardiomyopathy treated with 
tafamidis, a transthyretin stabilising agent. Tafamidis is expected to be available for clinical use in the near future.

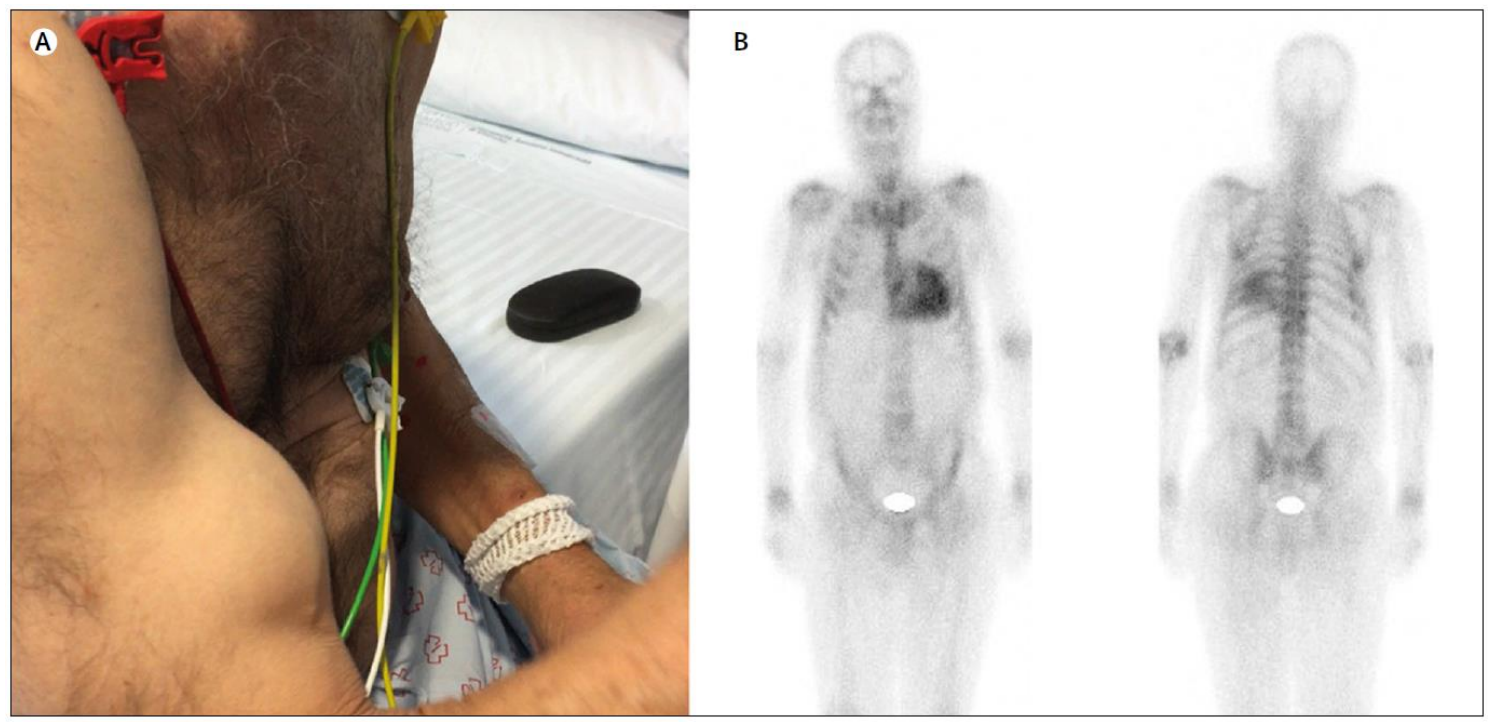

Figure: Popeye's sign in wild-type transthyretin amyloidosis

(A) Popeye's sign indicating rupture of the proximal biceps tendon. (B) ${ }^{99 \mathrm{~m}}$ Technetium-3,3-diphosphono-1,2-propanodicarboxylic acid scintigraphy images from both front and back show grade 3 myocardial uptake.

Contributors We were all involved in the clinical care of the patient, and the writing and approval of the manuscript. Written consent for publication was obtained from the patient.

Acknowledgements This work was co-funded by the European Regional Development Fund. 